\section{A Generalization of Some Existence Results on Orthogonal Designs for STBCs}

\author{
Md. Zafar Ali Khan, Student Member, IEEE, and \\ B. Sundar Rajan, Senior Member, IEEE
}

\begin{abstract}
It is shown that two theorems regarding the existence of generalized linear processing orthogonal designs (GLPODs) by Tarokh, Jafarkhani, and Calderbank (in "Space-time block codes from orthogonal designs," IEEE Trans. Inform. Theory, vol. 45, pp. 1456-1467, July 1999) are valid under more general conditions than for which they have been stated and proved.
\end{abstract}

Index Terms-Fast maximum-likelihood (ML) decoding, orthogonal designs, space-time block codes, transmit diversity.

\section{INTRODUCTION}

A generalized linear processing complex orthogonal design (GLPCOD) [1], [2] in $k$ complex indeterminates $x_{1}, x_{2}, \ldots, x_{k}$ of size $n$ and rate $\mathcal{R}=k / p, p \geq n$ is a $p \times n$ matrix $\mathcal{E}$, such that

- the entries of $\mathcal{E}$ are complex linear combinations of $0, \pm x_{i}, i=$ $1, \ldots, k$ and their conjugates;

- $\mathcal{E}^{H} \mathcal{E}=\boldsymbol{D}$, where $\mathcal{E}^{H}$ is the Hermitian (conjugate transpose) of $\mathcal{E}$ and $D$ is a diagonal matrix with the $(i, i)$ th diagonal element of the form

$$
l_{1}^{(i)}\left|x_{1}\right|^{2}+l_{2}^{(i)}\left|x_{2}\right|^{2}+\cdots+l_{k}^{(i)}\left|x_{k}\right|^{2}
$$

where $l_{j}^{(i)}, i=1,2, \ldots, n, j=1,2, \ldots, k$ are strictly positive numbers and for all values of $i$

$$
l_{1}^{(i)}=l_{2}^{(i)}=\cdots=l_{k}^{(i)} .
$$

The condition given by (2), which we will henceforth refer as equalweights condition has been introduced in [2] as a correction to [1]. If $k=n=p$, then $\mathcal{E}$ is called a linear processing complex orthogonal design (LPCOD). Furthermore, when the entries are only from $\left\{ \pm x_{1}, \pm x_{2}, \ldots, \pm x_{k}\right\}$, their conjugates and multiples of $\boldsymbol{j}$, where $\boldsymbol{j}=$ $\sqrt{-1}$, then $\mathcal{E}$ is called a complex orthogonal design (COD). When the entries of $\mathcal{E}$ are real variables and real linear combinations of these variables, it is called a generalized linear processing real orthogonal designs (GLPROD). A GLPROD satisfying $k=n=p$ is called a linear processing real orthogonal design (LPROD) and, in addition, if the entries are only from $\left\{ \pm x_{1}, \pm x_{2}, \ldots, \pm x_{k}\right\}$, then it is called a real orthogonal designs (ROD).

The existence of orthogonal designs (ODs) is of fundamental importance in the theory of space-time block codes [1]. In this regard, the paper [1] presents four theorems (Theorems 3.4.1, 4.1.1, 5.4.1, and 5.5.1): Theorem 3.4.1 deals with RODs, Theorem 5.4.1 with CODs,

Manuscript received February 21, 2003; revised May 25, 2003. This work was supported in part by the DRDO-IISc Program on Mathematical Engineering through a grant to B. S. Rajan.

M. Z. A. Khan was with the Department of Electrical Communication Engineering, Indian Institute of Science, Bangalore-560012, India. He is now with Insilica Semiconductors India Ltd., Bangalore-560 012, India (e-mail: zafar@protocol.ece.iisc.ernet.in).

B. S. Rajan is with the Department of Electrical Communication Engineering, Indian Institute of Science, Bangalore-560012, India (e-mail:bsrajan@ ece.iisc.ernet.in).

Communicated by K. Abdel-Ghaffar, Associate Editor for Coding Theory.

Digital Object Identifier 10.1109/TIT.2003.821969 and Theorems 4.1.1 and 5.5.1 with GLPROD and GLPCOD, respectively. The proof is given only for Theorem 3.4.1 and the remaining three theorems are stated with the remark that the proofs are similar to that of Theorem 3.4.1.

In this correspondence, we show that in case of square GLPCODs of square size, i.e., $n=p$, Theorems 3.4.1 and 5.4.1 of [1] are valid without the equal-weights condition in the definition of GLPCODs. Notice that the number of variables $k$ need not be equal to $n=p$.

\section{THEOREM 3.4.1 OF [1] REVISITED}

We begin by analyzing the proof of Theorem 3.4.1 given in [1], which is for real orthogonal designs, without assuming the equal-weights condition.

Theorem 1 ([1, Theorem 3.4.1]): A linear processing orthogonal design, $\mathcal{E}$ in variables $x_{1}, x_{2}, \ldots, x_{n}$ exists iff there exists a linear processing orthogonal design $\mathcal{L}$, such that

$$
\mathcal{L}^{T} \mathcal{L}=\mathcal{L L}^{T}=\left(x_{1}^{2}+x_{2}^{2}+\cdots+x_{n}^{2}\right) I .
$$

The proof given in [1] is as follows.

Let $\mathcal{E}=x_{1} A_{1}+\cdots+x_{n} A_{n}$ be a linear processing orthogonal design and let

$$
\mathcal{E}^{T} \mathcal{E}=x_{1}^{2} \mathcal{D}_{1}+\cdots+x_{n}^{2} \mathcal{D}_{n}
$$

where the matrices $\mathcal{D}_{i}$ are diagonal and of full rank. Then, it follows that

$$
\begin{array}{ll}
A_{i}^{T} A_{i}=\mathcal{D}_{i}, & i=1, \ldots, n \\
A_{i}^{T} A_{j}=-A_{j}^{T} A_{i}, & 1 \leq i \neq j \leq n
\end{array}
$$

where $\mathcal{D}_{i}$ is a full-rank diagonal matrix with positive diagonal entries. Let $\mathcal{D}_{i}^{1 / 2}$ be the diagonal matrix having the property that $\mathcal{D}_{i}^{1 / 2} \mathcal{D}_{i}^{1 / 2}=$ $\mathcal{D}_{i}$. Define

$$
U_{i}=A_{i} \mathcal{D}_{i}^{-1 / 2}
$$

Then the matrices $U_{i}$ satisfy the following properties:

$$
\begin{array}{ll}
U_{i}^{T} U_{i}=I, & i=1, \ldots, n \\
U_{i}^{T} U_{j}=-U_{j}^{T} U_{i}, & 1 \leq i \neq j \leq n .
\end{array}
$$

After this step, the proof is completed in [1] stating that $\mathcal{L}=x_{1} U_{1}+$ $x_{2} U_{2}+\cdots+x_{n} U_{n}$ is a linear processing orthogonal design satisfying (3). Substituting from (7) in (9) one gets

$$
\mathcal{D}_{i}^{-1 / 2} A_{i}^{T} A_{j} \mathcal{D}_{j}^{-1 / 2}=-\mathcal{D}_{j}^{-1 / 2} A_{j}^{T} A_{i} \mathcal{D}_{i}^{-1 / 2}, \quad 1 \leq i \neq j \leq n
$$

which is same as

$$
\mathcal{D}_{i}^{-1 / 2} A_{i}^{T} A_{j} \mathcal{D}_{j}^{-1 / 2}=\mathcal{D}_{j}^{-1 / 2} A_{i}^{T} A_{j} \mathcal{D}_{i}^{-1 / 2}, \quad 1 \leq i \neq j \leq n .
$$

If the equal-weights condition is included in the definition of GLPCOD then clearly (11) is satisfied and the proof is complete. However, even without the equal-weights condition, (9) is valid as shown in the following section. To be specific, in the following section it is shown that for square designs the set of $\mathcal{D}_{i}$ 's in (4) satisfy (10) without the equal-weights condition included in the definition of GLPROD. This is done by way of proving a generalization of Theorems 3.4.1 and 5.4.1 of [1] for all square designs real or complex. 


\section{A GeNERALIZATION OF THEOREMS 3.4.1 AND 5.4.1 OF [1]}

In this section, we prove a generalization of Theorems 3.4.1 and 5.4.1 of [1]. Note that Theorems 3.4.1 and 5.4.1 of [1] assume $n=p=k$ whereas our theorem assumes $n=p$ and there is no restriction on $k$.

Theorem 2: With the equal-weights condition removed from the definition of GLPCODs, an $n \times n$ square (GLPCOD), $\mathcal{E}_{c}$ in variables $x_{1}, \ldots, x_{k}$ exists iff there exists a GLPCOD $\mathcal{L}_{c}$ such that

$$
\mathcal{L}_{c}^{H} \mathcal{L}_{c}=\left(\left|x_{1}\right|^{2}+\cdots+\left|x_{k}\right|^{2}\right) I .
$$

Proof: Let $\mathcal{E}_{c}=\sum_{i=1}^{k} x_{i I} A_{2 i-1}+x_{i Q} A_{2 i}$ where $x_{i}=x_{i I}+\boldsymbol{j} x_{i Q}$. The weight matrices $\left\{A_{i}\right\}$ satisfy

$$
\begin{aligned}
A_{i}^{H} A_{i} & =\mathcal{D}_{i}, & & i=1, \ldots, 2 k \\
A_{j}^{H} A_{i}+A_{i}^{H} A_{j} & =0, & & 1 \leq i \neq j \leq 2 k .
\end{aligned}
$$

It is important to observe that $\mathcal{D}_{i}$ is a diagonal and full-rank matrix for all $i$. Define $B_{i}=A_{i} \mathcal{D}_{i}^{-1 / 2}$ and $\mathcal{L}_{c}=\sum_{i=1}^{k} x_{i I} B_{2 i-1}+x_{i Q} B_{2 i}$. Then the design $\mathcal{L}_{c}$ satisfies (12) iff the matrices $B_{i}$ satisfy

$$
\begin{aligned}
B_{i}^{H} B_{i} & =I_{N}, & & i=1, \ldots, 2 k \\
B_{j}^{H} B_{i}+B_{i}^{H} B_{j} & =0, & & 1 \leq i \neq j \leq 2 k .
\end{aligned}
$$

Substituting $B_{i}=A_{i} \mathcal{D}_{i}^{-1 / 2}$, while (15) is always satisfied, (16) is satisfied iff

$\mathcal{D}_{i}^{-1 / 2} A_{i}^{H} A_{j} \mathcal{D}_{j}^{-1 / 2}=-\mathcal{D}_{j}^{-1 / 2} A_{j}^{H} A_{i} \mathcal{D}_{i}^{-1 / 2}, \quad 1 \leq i \neq j \leq 2 k$

Notice that (17) reduces to (10) for real orthogonal designs and with $k=n$. In what follows we show that for square designs, (17) is satisfied without the equal-weights condition in the definition of GLPCODs.

Define $B_{i}^{(l)}=\mathcal{D}_{l}^{-1 / 2} A_{l}^{H} A_{i} \mathcal{D}_{l}^{-1 / 2}$ for $1 \leq l, i \leq 2 k$. Then $B_{l}^{(l)}=I_{n}$ and

$$
\begin{array}{ll}
{\left[B_{i}^{(l)}\right]^{H}=-B_{i}^{(l)}} & \\
{\left[B_{i}^{(l)}\right]^{2}=-\mathcal{D}_{l}^{-1} \mathcal{D}_{i} \triangleq \hat{\mathcal{D}}_{i}^{(l)},} & 1 \leq l \neq i \leq 2 k \\
B_{j}^{(l)} B_{i}^{(l)}+B_{i}^{(l)} B_{j}^{(l)}=0, & 1 \leq i \neq l \neq j \leq 2 k
\end{array}
$$

where we have used the fact that $\mathcal{D}_{l}^{-1} A_{l}^{H}$ is the inverse of $A_{l}$, so $A_{l} \mathcal{D}_{l}^{-1} A_{l}^{H}=I$ (which is true only for square $A_{l}$ ). Now the inverse of $B_{i}^{(l)}, 1 \leq i \neq l \leq 2 k$ is $B_{i}^{(l)}\left[\hat{\mathcal{D}}_{i}^{(l)}\right]^{-1}$ and also $\left[\hat{\mathcal{D}}_{i}^{(l)}\right]^{-1} B_{i}^{(l)}$ which can be verified by multiplying with $B_{i}^{(l)}$ and then using (18). Since the inverse is unique, we have

$$
B_{i}^{(l)}\left[\hat{\mathcal{D}}_{i}^{(l)}\right]^{-1}=\left[\hat{\mathcal{D}}_{i}^{(l)}\right]^{-1} B_{i}^{(l)}, \quad 1 \leq i \neq l \leq 2 k .
$$

The $(r, m)$ th entry, where $(1 \leq r, m \leq n)$, of $B_{i}^{(l)}\left[\hat{\mathcal{D}}_{i}^{(l)}\right]^{-1}$ is $b_{r, m}^{(i)} d_{m}^{(i)}$ where $b_{r, m}^{(i)}$ is the $(r, m)$ th entry of $B_{i}^{(l)}$ and $d_{m}^{(i)}$ is the $m$ th entry of $\left[\hat{\mathcal{D}}_{i}^{(l)}\right]^{-1}$. Similarly, the $(r, m)$ th entry of $\left[\hat{\mathcal{D}}_{i}^{(l)}\right]^{-1} B_{i}^{(l)}$ is $d_{r}^{(i)} b_{r, m}^{(i)}$. Equating the $(r, m)$ th entries on both sides of (21), we have

$$
b_{r, m}^{(i)} d_{m}^{(i)}=d_{r}^{(i)} b_{r, m}^{(i)}, \quad \forall r, m .
$$

If $b_{r, m}^{(i)} \neq 0$ then $d_{m}^{(i)}=d_{r}^{(i)}$, otherwise, both sides of (22) are 0 . In either case, we can multiply the left-hand side term by $\left[d_{m}^{(i)}\right]^{-1 / 2}$ and the right-hand side term by $\left[d_{r}^{(i)}\right]^{-1 / 2}$ to obtain

$$
\begin{aligned}
b_{r, m}^{(i)}\left[d_{m}^{(i)}\right]^{1 / 2} & =\left[d_{r}^{(i)}\right]^{1 / 2} b_{r, m}^{(i)}, & & \forall r, m \\
\Rightarrow B_{i}^{(l)}\left[\hat{\mathcal{D}}_{i}^{(l)}\right]^{-1 / 2} & =\left[\hat{\mathcal{D}}_{i}^{(l)}\right]^{-1 / 2} B_{i}^{(l)}, & & 1 \leq i \neq l \leq 2 k .
\end{aligned}
$$

Substituting the value of $B_{i}^{(l)}, \hat{\mathcal{D}}_{i}^{(l)}$ from (18) in (23) we have

$$
\begin{aligned}
& \mathcal{D}_{l}^{-1 / 2} A_{l}^{H} A_{i} \mathcal{D}_{l}^{-1 / 2}\left[-\mathcal{D}_{l}^{-1} \mathcal{D}_{i}\right]^{-1 / 2} \\
= & {\left[-\mathcal{D}_{l}^{-1} \mathcal{D}_{i}\right]^{-1 / 2} \mathcal{D}_{l}^{-1 / 2} A_{l}^{H} A_{i} \mathcal{D}_{l}^{-1 / 2} } \\
\Rightarrow & \mathcal{D}_{l}^{-1 / 2} A_{l}^{H} A_{i} \mathcal{D}_{i}^{-1 / 2}=\mathcal{D}_{i}^{-1 / 2} A_{l}^{H} A_{i} \mathcal{D}_{l}^{-1 / 2} \\
\Rightarrow & \mathcal{D}_{l}^{-1 / 2} A_{l}^{H} A_{i} \mathcal{D}_{i}^{-1 / 2}=-\mathcal{D}_{i}^{-1 / 2} A_{i}^{H} A_{l} \mathcal{D}_{l}^{-1 / 2}
\end{aligned}
$$

which is the same as (17) and the proof is complete.

When $k=n$, Theorem 2 reduces to Theorem 5.4.1 of [1]. Similarly, when $x_{i}$ 's are real, the weight matrices $A_{i}$ are real matrices and $k=n$. Then Theorem 2 includes Theorem 3.4.1 of [1]. The arguments of this correspondence cannot be used to prove Theorems 4.1.1 and 5.5.1 in [1] without the equal-weights condition. Indeed, the two designs presented in [3] show that Theorems 4.1.1 and 5.5.1 of [1] are not valid without the equal-weights condition.

The results of [4] are for square designs satisfying the condition (12). By virtue of Theorem 2, the results of [4], [1] are valid for all square designs without the equal-weights conditions and hence we have the following corollary.

Corollary 3: Let $N=2^{a} b$ where $b$ is an odd integer and $a=4 c+d$, where $0 \leq d<c$ and $c \geq 0$. The maximal rate of size $N$ square GLPROD without the equal-weights condition satisfied is $\frac{8 c+2^{d}}{N}$ and of size $N$ square GLPCOD without the equal-weights condition satisfied is $\frac{a+1}{N}$.

\section{ACKNOWLEDGMENT}

The authors gratefully acknowledge Weifeng Su for pointing out a mistake in the proof of the main theorem in an earlier version of this correspondence and thank Kiran T. and V. Shashidhar for useful discussions on the subject matter. We thank the referees for their comments which helped to improve the clarity of the correspondence.

\section{REFERENCES}

[1] V. Tarokh, H. Jafarkhani, and A. R. Calderbank, "Space-time block codes from orthogonal designs," IEEE Trans. Inform. Theory, vol. 45, pp. 1456-1467, July 1999.

[2] —, "Correction to 'Space-time block codes from orthogonal designs'," IEEE Trans. Inform. Theory, vol. 46, p. 314, Jan. 2000.

[3] W. Su and X.-G. Xia, "Two generalized complex orthogonal space-time block codes of rates $7 / 11$ and $3 / 5$ for 5 and 6 transmit antennas," IEEE Trans. Inform. Theory, vol. 49, pp. 313-316, Jan. 2003.

[4] O. Tirkkonen and A. Hottinen, "Square-matrix embeddable space-time block codes for complex signal constellations," IEEE Trans. Inform. Theory, vol. 48, pp. 384-395, Feb. 2002. 